\title{
3-Benzoylisoxazolines by 1,3-Dipolar Cycloaddition: Chloramine-T-Catalyzed Condensation of $\alpha$-Nitroketones with Dipolarophiles
}

\author{
Xinhui Pan ${ }^{1,2, *,+}$, Xiaobing Xin ${ }^{2,3,+}$, Ying Mao ${ }^{4,+}$, Xin Li ${ }^{2}$, Yanan Zhao ${ }^{2} \mathbb{D}$, Yidi Liu ${ }^{2}$, Ke Zhang ${ }^{2}$, Xiaoda Yang ${ }^{1,2}$ \\ and Jinhui Wang ${ }^{2, *}$ \\ 1 Stake Key Laboratory of Natural and Biomimetic Drugs, Department of Chemical Biology, \\ School of Pharmaceutical Sciences, Peking University, Beijing 100191, China; xyang@bjmu.edu.cn \\ 2 Key Laboratory of Xinjiang Phytomedicine Resource and Utilisation, Ministry of Education, \\ School of Pharmaceutical Sciences, Shihezi University, Shihezi 832002, China; 18699300391@163.com (X.X.); \\ lixin@stu.shzu.edu.cn (X.L.); zynhyl@stu.shzu.edu.cn (Y.Z.); lyd@stu.shzu.edu.cn (Y.L.); \\ xjzk1984@163.com (K.Z.) \\ 3 Department of Pharmacology, Houbo College of Xinjiang Medical University, Karamay 834000, China \\ 4 School of Chemistry and Chemical Engineering, Shandong University, Jinan 250100, China; \\ maoying1984@163.com \\ * Correspondence: panxhshzu@shzu.edu.cn (X.P.); wangjinhui@hrbmu.edu.cn (J.W.) \\ $+\quad$ These authors contributed equally.
}

\begin{abstract}
In this study, 3-benzoylisoxazolines were synthesized by reacting alkenes with various $\alpha$ nitroketones using chloramine-T as the base. The scope of $\alpha$-nitroketones and alkenes is extensive, including different alkenes and alkynes to form various isoxazolines and isoxazoles. The use of chloramine-T, as the low-cost, easily handled, moderate base for 1,3-dipolar cycloaddition is attractive.
\end{abstract}

Keywords: isoxazolines; $\alpha$-nitroketones; alkenes; chloramine-T; 1,3-dipolar cycloaddition

\section{Introduction}

Isoxazoline derivatives have been demonstrated to exhibit a variety of biological and pharmacological activities, such as antithrombotic effects, insect growth regulation, immunopotentiation and anticancer activities (Figure 1) [1-5]. In organic synthesis, isoxazolines are also useful intermediates. For instance, they can be converted into different critical synthetic units, such as $\beta$-hydroxy ketones [6], $\gamma$-amino alcohols [7], $\alpha, \beta$-unsaturated ketones [8] and $\beta$-hydroxy nitriles [9]. Carreira et al. and Lee et al. [10-12] used isoxazolines in the total synthesis of natural products (Figure 2).

Previous studies described various methods for synthesizing isoxazolines, namely, the 1,3-dipolar cycloaddition of dipolarophiles [13] (alkynes, alkenes) with nitrile oxides from aldoximes [14] or $\alpha$-nitroketones. In the case of $\alpha$-nitroketones, nitrile oxides are prepared by dehydration with an acid (such as sulfuric acid [15], $p$-toluenesulfonic acid [16,17] or polyphosphoric acid-silica (PPA/SiO $)$ [18]) or a base (such as N-methylimidazole [19], 1,4-diazabicyclo [2.2.2] octane [20] and copper (II) acetate/N-methylpiperidine [21]) as the catalytic systems.

Moderate to good reaction efficiency can be obtained by utilizing these acidic or basic catalytic systems. The majority of the reactions undergo 1,3-dipolar cycloadditions with different $\alpha$-nitroketones to obtain various isoxazoles. However, it is reported that 1,3-dipolar cycloadditions that start from nitroalkanes bearing a carbonyl group do not proceed smoothly and thus, generate the desired products in low yields. Tsubaki et al. [22] recently developed nitrile oxide cycloaddition reactions between nitrile oxides derived from O-alkyloxime-substituted nitroalkanes and various alkenes to construct 2-isoxazolines with electron-withdrawing groups. Although this method proceeds smoothly with nitroalkanes 
and various alkenes, it requires the preparation of O-alkyloxime-substituted nitroalkanes as precursors. To simplify this reaction, we tested several other bases and identified chloramine-T a commercially available agent, to give superior results [23]. We found that chloramine salts showed highly attractive practical characteristics: easy and amenable preparation at large scales, nontoxic by-products, excellent reactivity and high stability to air and heat. In this study, we successfully developed a chloramine-T catalytic system for 1,3-dipolar cycloaddition of dipolarophiles with $\alpha$-nitroketones. To the best of our knowledge, it is the first time that chloramine-T has been used in 1,3-dipolar cycloaddition reactions to obtain isoxazolines from $\alpha$-nitroketones.<smiles>CC(=O)OCNC(=N)c1ccc(C2=NOC(CC(=O)NC[C@H](NC(=O)OCc3ccccc3)C(C)=O)C2)cc1</smiles>

antithrombotic agents<smiles>COc1ccc(C2CC(c3cc(OC)c(OCOc4cc(C5CC(c6cc(OC)c(OC)c(OC)c6)NC5=O)ccc4OC)c(OC)c3)=NO2)cc1OC</smiles>

immunopotentiating activity

anticancer activity

Figure 1. Examples of important isoxazolines.

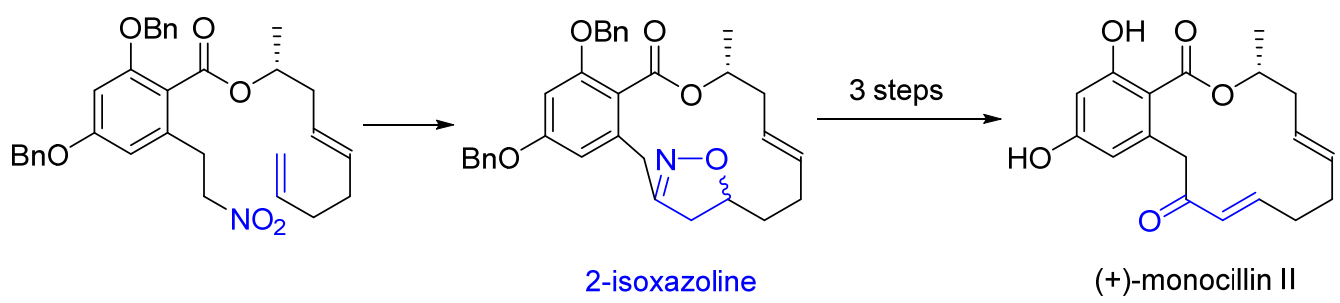

Figure 2. Application of 2-isoxazoline as a key building block.

\section{Results and Discussion}

Benzoylnitromethane 1a (1 equiv) was reacted with allylbenzene $2 a$ (5 equiv) in the presence of various bases in acetonitrile at $80^{\circ} \mathrm{C}$. The results are summarized in Table 1 (entries 1-6). No reaction occurred in the base-free system. Chloramine-T was the best among the bases tested herein. It is conceivable that 0.5 equivalents of chloramine- $T$ can provide a better yield (Table 1, entries 6-9). According to the results, a few solvents were screened for optimization. Cycloaddition was found to be reliant on the solvent. Although $\mathrm{H}_{2} \mathrm{O}$ inhibited the cycloaddition, the reaction worked in DMSO and DMF, while $\mathrm{CH}_{3} \mathrm{CN}$ as the solvent provided the best results (Table 1, entries 10-13), producing 3a with a yield of $77 \%$. Increasing the temperature to $90^{\circ} \mathrm{C}$ or lowering it to $60^{\circ} \mathrm{C}$ resulted in a lesser yield (Table 1, entries 14 and 15). Thus, the reaction in optimal conditions was conducted at $80^{\circ} \mathrm{C}$ for $18 \mathrm{~h}$ with 0.5 equiv of chloramine-T in the presence of acetonitrile as the solvent; and $3 \mathbf{a}$ was obtained with a yield of $77 \%$. 
Table 1. Reaction condition optimization ${ }^{\mathrm{a}}$.

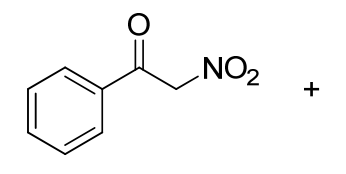

$1 \mathrm{a}$<smiles>C=CCc1ccccc1</smiles>

$2 a$

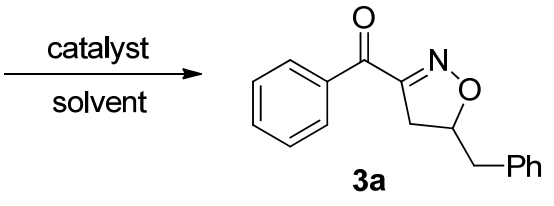

3a

\begin{tabular}{|c|c|c|c|}
\hline Entry & Catalyst & Amount of Base (mol) & Yield $^{b}(\%)$ of $3 a$ \\
\hline 1 & - & - & $<5$ \\
\hline 2 & Imidazole & 0.5 & 43 \\
\hline 3 & 4-Dimethylaminopyridine & 0.5 & 45 \\
\hline 4 & Triethylamine & 0.5 & 55 \\
\hline 5 & $\begin{array}{c}\mathrm{N}, \mathrm{N}, \mathrm{N}^{\prime}, \mathrm{N}^{\prime}- \\
\text { Tetramethylethylenediamine }\end{array}$ & 0.5 & 48 \\
\hline 6 & Chloramine- $\mathrm{T}$ & 0.5 & 77 \\
\hline 7 & Chloramine- $\mathrm{T}$ & 1.0 & 52 \\
\hline 8 & Chloramine- $\mathrm{T}$ & 0.25 & 68 \\
\hline 9 & Chloramine- $\mathrm{T}$ & 0.1 & 65 \\
\hline $10^{c}$ & Chloramine- $\mathrm{T}$ & 0.5 & 46 \\
\hline $11^{\mathrm{d}}$ & Chloramine- $\mathrm{T}$ & 0.5 & 40 \\
\hline $12^{\mathrm{e}}$ & Chloramine- $\mathrm{T}$ & 0.5 & 11 \\
\hline $13^{\mathrm{f}}$ & Chloramine- $\mathrm{T}$ & 0.5 & 36 \\
\hline $14^{\mathrm{g}}$ & Chloramine- $\mathrm{T}$ & 0.5 & 70 \\
\hline $15^{\mathrm{h}}$ & Chloramine- $\mathrm{T}$ & 0.5 & 59 \\
\hline
\end{tabular}

a General conditions: 1a $(0.125 \mathrm{mmol}), \mathbf{2 a}(0.625 \mathrm{mmol})$, chloramine- $\mathrm{T}(0.0625 \mathrm{mmol}), \mathrm{CH}_{3} \mathrm{CN}(0.2 \mathrm{~mL})$ at $80{ }^{\circ} \mathrm{C}$ for $18 \mathrm{~h}$, unless stated otherwise. ${ }^{\mathrm{b}}$ Isolated yield. ${ }^{\mathrm{c}} \mathrm{DMF}$ as the solvent. ${ }^{\mathrm{d}} \mathrm{DMSO}$ as the solvent. ${ }^{\mathrm{e}} \mathrm{H}_{2} \mathrm{O}$ as the solvent. ${ }^{\mathrm{f}} \mathrm{CH}_{3} \mathrm{NO}_{2}$ as the solvent. ${ }^{\mathrm{g}}$ Reaction at $90{ }^{\circ} \mathrm{C}$. ${ }^{\mathrm{h}}$ Reaction at $60{ }^{\circ} \mathrm{C}$.

Under the optimized reaction conditions, various substrates were subjected to 1,3dipolar cycloaddition (Scheme 1). Several electronically varied $\alpha$-nitroketones were subjected to cycloaddition. Efficiency was considered as the sensitivity of cycloaddition to electronic substituents. Electron-deficient $\alpha$-nitroketones (3b-3d, Scheme 1) provided products in slightly better yields related to electron-rich $\alpha$-nitroketones (3e and $3 \mathbf{f}$, Scheme 1). Phenacyl nitro derivatives incorporating tert-butyl and phenyl at the para-position were also successful in the cycloaddition (3g and $3 \mathbf{h}$, Scheme 1). Simple nitroketones were efficiently coupled with allylbenzene ( $3 \mathbf{i}$, Scheme 1$)$.

Next, we investigated the scope of the alkenes in Scheme 2. Alkene alternative with contrasting and electronically varied substituents reacted with benzoylnitromethane 1a smoothly under the standard conditions to obtain the desired products in excellent yields. Moreover, the electron-rich allylbenzenes and allylalkanes produced the product in good yields (5a-5e, Scheme 2). Similarly, the electron-deficient ones, such as allyl chloride, produced the product in excellent yields ( $5 f$, Scheme 2). Good yields were also obtained in the cycloaddition of cyclohexene (5g, Scheme 2$)$. 


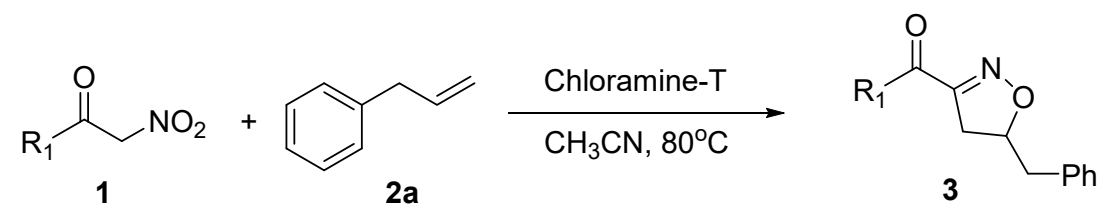<smiles>O=C(C1=NOC(Cc2ccccc2)C1)c1ccccc1</smiles><smiles>O=C(C1=NOC(Cc2ccccc2)C1)c1ccccc1Br</smiles><smiles>O=C(C1=NOC(Cc2ccccc2)C1)c1cccc(Br)c1</smiles><smiles>O=C(C1=NOC(Cc2ccccc2)C1)c1ccc(Br)cc1</smiles><smiles>Cc1ccc(C(=O)C2=NOC(Cc3ccccc3)C2)cc1</smiles>
3e $68 \%$<smiles>COc1ccc(C(=O)C2=NOC(Cc3ccccc3)C2)cc1</smiles><smiles>CC(C)(C)c1ccc(C(=O)C2=NOC(Cc3ccccc3)C2)cc1</smiles><smiles>O=C(C1=NOC(Cc2ccccc2)C1)c1ccc(-c2ccccc2)cc1</smiles><smiles>COC(=O)C1=NOC(Cc2ccccc2)C1</smiles>

3i $30 \%$

Scheme 1. Scope of cycloaddition.<smiles>O=C(C[N+](=O)[O-])c1ccccc1</smiles>

1a<smiles>CON=C(CC(Cc1ccccc1C)C(=O)c1ccccc1)C(=O)[18O]c1ccccc1</smiles><smiles>COc1ccc(CC2CC(C(=O)c3ccccc3)=NO2)cc1</smiles>

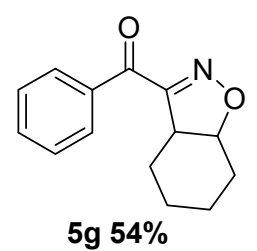

Scheme 2. Scope of alkenes.<smiles>[R]C=CC(=O)N(C)OC</smiles>

4<smiles>Cc1cccc(CC2CC(C(=O)c3ccccc3)=NO2)c1</smiles>

5b $66 \%$<smiles>CCC1CC(C(=O)c2ccccc2)=NO1</smiles>

5e $89 \%$<smiles>[R3]C1ON=C(C(=O)c2ccccc2)C1[R]</smiles><smiles>Cc1ccc(CC2CC(C(=O)c3ccccc3)=NO2)cc1</smiles>

5 c $64 \%$<smiles>O=C(C1=NOC(CCl)C1)c1ccccc1</smiles>

5f $83 \%$ 
Finally, 1a was reacted with 1-hexyne in the presence of chloramine-T in acetonitrile at $70{ }^{\circ} \mathrm{C}$ for $18 \mathrm{~h}$. Isoxazoles $7 \mathbf{a}$ and $7 \mathbf{b}$ were obtained with a yield of $68 \%$ and $64 \%$ (Scheme 3). This result demonstrated that this reaction is also suitable for cycloadditions to form isoxazoles.<smiles>O=C(C[N+](=O)[O-])c1ccccc1</smiles>

$1 \mathrm{a}$

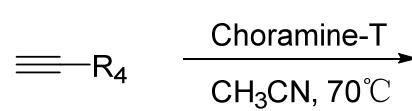

6<smiles>[R4]c1cc(C(=O)c2ccccc2)no1</smiles>

7

$7 \mathrm{a}, \mathrm{R}_{4}=\mathrm{COOC}_{2} \mathrm{H}_{5}, 68 \%$

$7 b, \mathrm{R}_{4}=\mathrm{n}-\mathrm{C}_{4} \mathrm{H}_{9}, 64 \%$

Scheme 3. The reaction of $1 \mathrm{a}$ with 6 in the presence of chloramine- $\mathrm{T}$ in $\mathrm{CH}_{3} \mathrm{CN}$.

A possible mechanism for the reaction is shown in Scheme 4. In acetonitrile, the ion pair $\mathbf{9}$, which is formed between nitronate $\mathbf{1}$ and the protonated base $\mathbf{8}$, undergoes cycloaddition with dipolarophile 2 to obtain intermediate 10. Subsequently, the ion pair intermediate adduct $\mathbf{1 0}$ releases chloramine-T $\mathbf{8}$ to produce the 2-hydroxyoxazolidine 11, which is then dehydrated to give the product 3 . Finally, another nitronate 1 reacts with chloramine-T 8 to obtain the new ion pair intermediate 9.

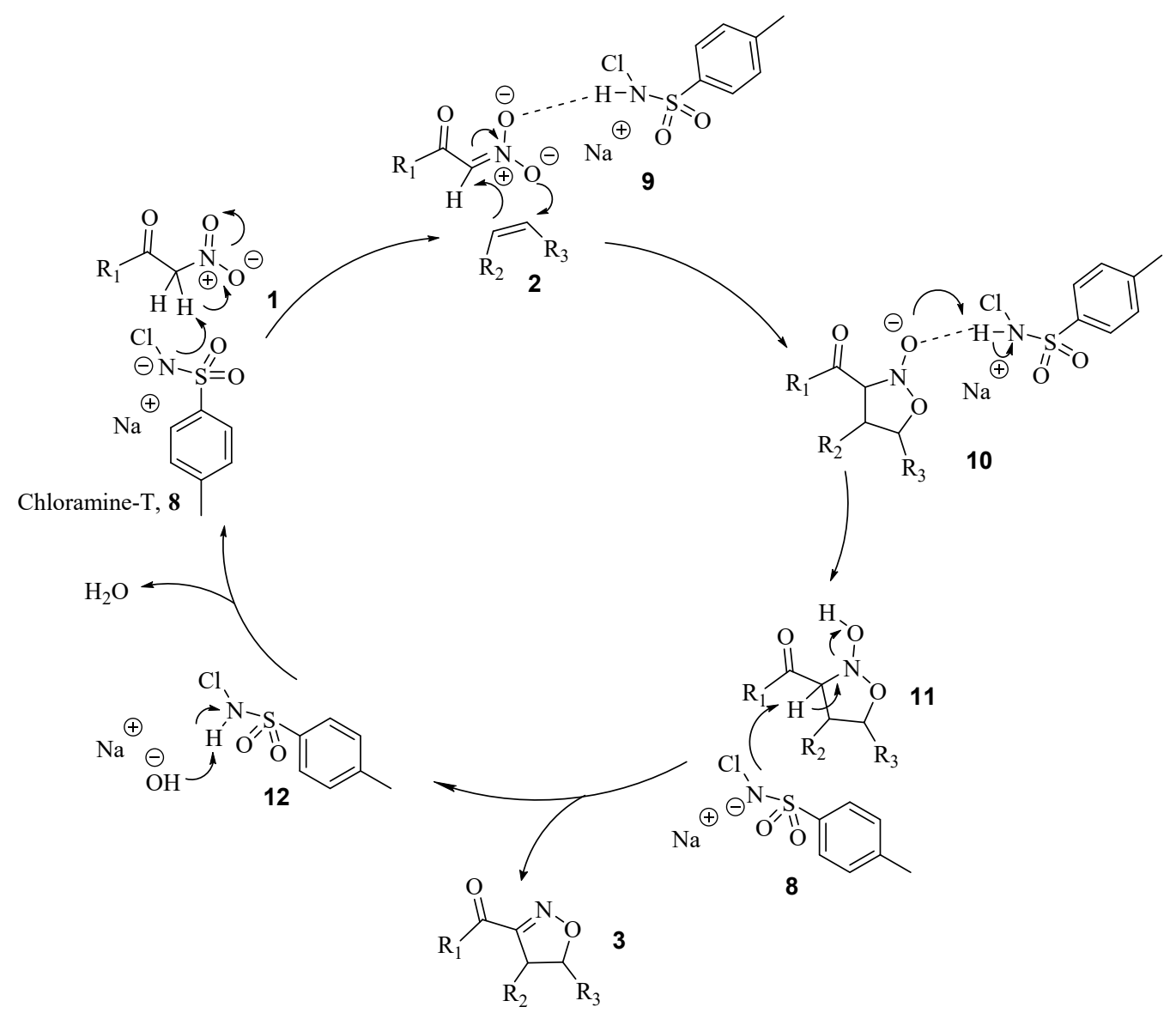

Scheme 4. A plausible mechanism for the condensation of nitro compounds and dipolarophiles. 


\section{Experimental Section}

\subsection{General Experimental Methods}

The structures of produced compounds were firmly confirmed by ${ }^{13} \mathrm{C}$ NMR and ${ }^{1} \mathrm{H}$ NMR spectra and supported by HRMS, IR data (see the Supplementary Materials).

${ }^{1} \mathrm{H}$ NMR $(400 \mathrm{MHz})$ and ${ }^{13} \mathrm{C}$ NMR $(101 \mathrm{MHz})$ were recorded at room temperature by using a DRX-400 spectrometer (Bruker, Germany) in $\mathrm{CDCl}_{3}$. Chemical shifts were given in parts per million (ppm) on the delta $(\delta)$ scale. The solvent peak was used as a reference value, for ${ }^{1} \mathrm{H}$ NMR: $\mathrm{CDCl}_{3} \delta 7.26$; for ${ }^{13} \mathrm{C} \mathrm{NMR}: \mathrm{CDCl}_{3}$ at $77.16 \mathrm{ppm}$. IR spectra were recorded using an Avatar 360 FT-IR ESP spectrometer Nicolet (Waltham, MA, USA) at room temperature. HR-ESI-MS spectra were acquired using an Agilent 6210 ESI/TOF mass spectrometer (Agilent Technologies, Santa Clara, CA, USA). Analytical TLC was run on silica gel plates (GF254, Yantai Institute of Chemical Technology, Yantai, China). Spots on the plates were observed under UV light. Column chromatography was performed on silica gels (200 300 mesh and 300-400 mesh; Qingdao Marine Chemical Factory, Qingdao, China). Super-dry solvent $\mathrm{CH}_{3} \mathrm{CN}, \mathrm{DMSO}$ and DMF were purchased from Aldrich and used as supplied. The $\alpha$-nitroketones were synthesized using the same method as reported in the literature [16].

\subsection{General Procedure for the Cycloaddition of Alkenes and $\alpha$-Nitroketones}

Chloramine-T ( $0.0625 \mathrm{mmol}, 0.5$ equiv) was added to a solution of $\mathbf{1}(0.125 \mathrm{mmol}$, 1 equiv) and 2 ( $0.625 \mathrm{mmol}, 5$ equiv) (or 4 ( $0.625 \mathrm{mmol}, 5$ equiv) or 6 ( $0.625 \mathrm{mmol}, 5$ equiv)) in $\mathrm{CH}_{3} \mathrm{CN}(0.2 \mathrm{~mL})$. The mixture was then stirred at $80^{\circ} \mathrm{C}$ until the starting material disappeared, as monitored by TLC. Subsequently, the mixture was directly purified by flash chromatography (with ethyl acetate/petroleum ether as the eluent) to obtain the desired product $(3,5$ or 7$)$.

\subsection{1. (5-Benzyl-4,5-dihydroisoxazol-3-yl)(phenyl)methanone (3a)}

The compound (with a yield of $77 \%$ ) was prepared following the general procedure described in Section 3.2. ${ }^{1} \mathrm{H}$ NMR $\left(400 \mathrm{MHz}, \mathrm{CDCl}_{3}\right) \delta 8.22-8.12(\mathrm{~m}, 2 \mathrm{H}), 7.63-7.57(\mathrm{~m}, 1 \mathrm{H})$, 7.51-7.44 (m, 2H), 7.38-7.33 (m, 2H), $7.28(\mathrm{~m}, 3 \mathrm{H}), 5.08(\mathrm{ddt}, J=10.8,7.9,6.3 \mathrm{~Hz}, 1 \mathrm{H})$, $3.37(\mathrm{dd}, J=17.6,10.8 \mathrm{~Hz}, 1 \mathrm{H}), 3.19-3.08(\mathrm{~m}, 2 \mathrm{H}), 2.98(\mathrm{dd}, J=14.0,6.5 \mathrm{~Hz}, 1 \mathrm{H})$; ${ }^{13} \mathrm{C}$ NMR $\left(101 \mathrm{MHz}, \mathrm{CDCl}_{3}\right) \delta$ 186.2, 157.5, 135.8, 135.6, 133.3, 130.1(2C), 129.3(2C), 128.5(2C), 128.1(2C), 126.8, 83.3, 40.7, 38.1; IR vmax 3033, 1654, 1581, 710, $672 \mathrm{~cm}^{-1}$; HRMS (EI) $\mathrm{m} / \mathrm{z}$ calcd for $\mathrm{C}_{17} \mathrm{H}_{16} \mathrm{NO}_{2}[\mathrm{M}+\mathrm{H}]^{+} 266.1176$, found 266.1178. These data are consistent with the data reported in the literature [11].

\subsection{2. (5-Benzyl-4,5-dihydroisoxazol-3-yl)(2-bromophenyl)methanone (3b)}

The compound was prepared following the general procedure. Yield $73 \% .{ }^{1} \mathrm{H}$ NMR $\left(400 \mathrm{MHz}, \mathrm{CDCl}_{3}\right) \delta 7.51(\mathrm{~d}, J=7.6 \mathrm{~Hz}, 1 \mathrm{H}), 7.29-7.25(\mathrm{~m}, 2 \mathrm{H}), 7.24(\mathrm{~m}, 2 \mathrm{H}), 7.21(\mathrm{~m}, 1 \mathrm{H})$, $7.18(\mathrm{~m}, 2 \mathrm{H}), 7.15(\mathrm{~m}, 1 \mathrm{H}), 5.05(\mathrm{ddt}, J=11.0,7.6,6.3 \mathrm{~Hz}, 1 \mathrm{H}), 3.22(\mathrm{dd}, J=17.5,10.9 \mathrm{~Hz}$, $1 \mathrm{H}), 3.04-2.94(\mathrm{~m}, 2 \mathrm{H}), 2.88(\mathrm{dd}, J=14.0,6.5 \mathrm{~Hz}, 1 \mathrm{H}) ;{ }^{13} \mathrm{C}$ NMR $(101 \mathrm{MHz}, \mathrm{CDCl} 3) \delta 189.2$, $158.1,139.2,135.8,133.4,132.1,129.8,129.7(2 \mathrm{C}), 128.8(2 \mathrm{C}), 127.2,127.1,120.0,85.2,41.0,36.7$; IR $v \max 3034,1680,1585,755,694 \mathrm{~cm}^{-1}$; HRMS (EI) $m / z$ calcd for $\mathrm{C}_{17} \mathrm{H}_{15} \mathrm{NO}_{2} \mathrm{Br}[\mathrm{M}+\mathrm{H}]^{+}$ 344.0281 , found 344.0279 .

\subsection{3. (5-Benzyl-4,5-dihydroisoxazol-3-yl)(3-bromophenyl)methanone (3c)}

The compound was prepared following the general procedure. Yield $67 \% .{ }^{1} \mathrm{H}$ NMR $\left(400 \mathrm{MHz}, \mathrm{CDCl}_{3}\right) \delta 8.24(\mathrm{~m}, 1 \mathrm{H}), 8.07(\mathrm{~m}, 1 \mathrm{H}), 7.70(\mathrm{~m}, 1 \mathrm{H}), 7.37-7.33(\mathrm{~m}, 2 \mathrm{H}), 7.32(\mathrm{~m}, 1 \mathrm{H})$, $7.27(\mathrm{~m}, 3 \mathrm{H}), 5.16-5.03(\mathrm{~m}, 1 \mathrm{H}), 3.34(\mathrm{dd}, J=17.6,10.9 \mathrm{~Hz}, 1 \mathrm{H}), 3.16-3.05(\mathrm{~m}, 2 \mathrm{H}), 2.98(\mathrm{dd}$, $J=14.0,6.4 \mathrm{~Hz}, 1 \mathrm{H}) ;{ }^{13} \mathrm{C}$ NMR $(101 \mathrm{MHz}, \mathrm{CDCl} 3) \delta$ 185.0, 157.6, 137.5, 136.4, 135.9, 133.2, 129.9, 129.6(2C), 128.9, 128.8(2C), 127.2, 122.6, 83.9, 40.9, 38.1; IR vmax 3038, 1691, 1616, 910, 811, $742 \mathrm{~cm}^{-1}$; HRMS (EI) $\mathrm{m} / z$ calcd for $\mathrm{C}_{17} \mathrm{H}_{15} \mathrm{NO}_{2} \mathrm{Br}[\mathrm{M}+\mathrm{H}]^{+} 344.0281$, found 344.0293 . 
3.2.4. (5-Benzyl-4,5-dihydroisoxazol-3-yl)(4-bromophenyl)methanone (3d)

The compound was prepared following the general procedure. Yield $72 \% .{ }^{1} \mathrm{H}$ NMR $\left(400 \mathrm{MHz}, \mathrm{CDCl}_{3}\right)^{1} \mathrm{H}$ NMR $\left(400 \mathrm{MHz}, \mathrm{CDCl}_{3}\right) \delta 8.04-7.97(\mathrm{~m}, 2 \mathrm{H}), 7.62-7.56(\mathrm{~m}, 2 \mathrm{H})$, $7.35-7.30(\mathrm{~m}, 2 \mathrm{H}), 7.25(\mathrm{~m}, 3 \mathrm{H}), 5.07(\mathrm{ddt}, J=10.9,7.9,6.3 \mathrm{~Hz}, 1 \mathrm{H}), 3.34(\mathrm{dd}, J=17.6,10.9 \mathrm{~Hz}$, 1H), 3.13-3.05 (m, 2H), $2.96(\mathrm{dd}, J=14.0,6.5 \mathrm{~Hz}, 1 \mathrm{H}) . ;{ }^{13} \mathrm{C} \mathrm{NMR}\left(101 \mathrm{MHz}, \mathrm{CDCl}_{3}\right) \delta 185.4$, 157.9, 136.1, 134.6, 132.0(2C), 131.9(2C), 129.7(2C), 129.2, 128.9(2C), 127.2, 83.9, 41.1, 38.3; IR vmax 3046, 1696, 1615, 801, 750, $686 \mathrm{~cm}^{-1}$; HRMS (EI) $m / z$ calcd for $\mathrm{C}_{17} \mathrm{H}_{15} \mathrm{NO}_{2} \mathrm{Br}$ $[\mathrm{M}+\mathrm{H}]^{+}$344.0281, found 344.0276.

\subsection{5. (5-Benzyl-4,5-dihydroisoxazol-3-yl)(p-tolyl)methanone (3e)}

The compound was prepared following the general procedure. Yield $68 \% .{ }^{1} \mathrm{H}$ NMR $\left(400 \mathrm{MHz}, \mathrm{CDCl}_{3}\right) \delta 8.08-8.03(\mathrm{~m}, 2 \mathrm{H}), 7.33(\mathrm{~m}, 2 \mathrm{H}), 7.28(\mathrm{~m}, 2 \mathrm{H}), 7.25(\mathrm{~m}, 3 \mathrm{H}), 5.05(\mathrm{ddt}$, mboxemph $=10.8,7.9,6.3 \mathrm{~Hz}, 1 \mathrm{H}), 3.35(\mathrm{dd}, J=17.6,10.8 \mathrm{~Hz}, 1 \mathrm{H}), 3.15-3.07(\mathrm{~m}, 2 \mathrm{H})$, $2.96(\mathrm{dd}, J=14.0,6.6 \mathrm{~Hz}, 1 \mathrm{H}), 2.42(\mathrm{~s}, 3 \mathrm{H}) ;{ }^{13} \mathrm{C} \mathrm{NMR}(101 \mathrm{MHz}, \mathrm{CDCl} 3) \delta 186.1,157.9$, 144.7, 136.2, 133.4, 130.6(2C), 129.6(2C), 129.2(2C), 128.8(2C), 127.1, 83.5, 41.1, 38.6, 21.9; IR $v \max 3023,2923,1650,1600,831,750,693 \mathrm{~cm}^{-1}$; HRMS (EI) $m / z$ calcd for $\mathrm{C}_{18} \mathrm{H}_{18} \mathrm{NO}_{2}$ $[\mathrm{M}+\mathrm{H}]^{+}$280.1332, found 280.1336.

\subsection{6. (5-Benzyl-4,5-dihydroisoxazol-3-yl)(4-methoxyphenyl)methanone (3f)}

The compound was prepared following the general procedure. Yield $54 \% .{ }^{1} \mathrm{H}$ NMR $\left(400 \mathrm{MHz}, \mathrm{CDCl}_{3}\right) \delta 8.23-8.15(\mathrm{~m}, 2 \mathrm{H}), 7.36-7.30(\mathrm{~m}, 2 \mathrm{H}), 7.29-7.25(\mathrm{~m}, 3 \mathrm{H}), 6.97-6.91(\mathrm{~m}, 2 \mathrm{H})$, $5.04(\mathrm{ddt}, J=10.8,7.9,6.4 \mathrm{~Hz}, 1 \mathrm{H}), 3.88(\mathrm{~s}, 3 \mathrm{H}), 3.36(\mathrm{dd}, J=17.6,10.8 \mathrm{~Hz}, 1 \mathrm{H}), 3.16-3.07(\mathrm{~m}, 2 \mathrm{H})$, $2.96(\mathrm{dd}, J=14.0,6.6 \mathrm{~Hz}, 1 \mathrm{H}) ;{ }^{13} \mathrm{C}$ NMR $(101 \mathrm{MHz}, \mathrm{CDCl} 3) \delta 184.7,164.2,157.9,136.3$, 132.9(2C), 129.6(2C), 128.8(2C), 127.1, 113.8(2C), 83.3, 55.7, 41.1, 38.8; IR vmax 3022, 2801, $1618,1531,802,719,676 \mathrm{~cm}^{-1}$; HRMS (EI) $\mathrm{m} / z$ calcd for $\mathrm{C}_{18} \mathrm{H}_{18} \mathrm{NO}_{3}[\mathrm{M}+\mathrm{H}]^{+} 296.1281$, found 296.1285 .

\subsection{7. (5-Benzyl-4,5-dihydroisoxazol-3-yl)(4-tert-butylphenyl)methanone (3g)}

The compound was prepared following the general procedure. Yield $63 \% .{ }^{1} \mathrm{H}$ NMR $\left(400 \mathrm{MHz}, \mathrm{CDCl}_{3}\right) \delta 8.10-8.05(\mathrm{~m}, 2 \mathrm{H}), 7.49-7.43(\mathrm{~m}, 2 \mathrm{H}), 7.34-7.23(\mathrm{~m}, 5 \mathrm{H}), 5.02(\mathrm{ddt}$, $J=10.8,7.9,6.3 \mathrm{~Hz}, 1 \mathrm{H}), 3.32(\mathrm{dd}, J=17.6,10.8 \mathrm{~Hz}, 1 \mathrm{H}), 3.14-3.04(\mathrm{~m}, 2 \mathrm{H}), 2.93(\mathrm{dd}$, $J=14.0,6.5 \mathrm{~Hz}, 1 \mathrm{H}), 1.34(\mathrm{~d}, J=4.4 \mathrm{~Hz}, 9 \mathrm{H}) ;{ }^{13} \mathrm{C} \mathrm{NMR}(101 \mathrm{MHz}, \mathrm{CDCl} 3) \delta$ 186.0, 157.8, 157.4, 136.2, 133.3, 130.3(2C), 129.5(2C), 128.7(2C), 126.9, 125.4(2C), 83.4, 40.9, 38.4, 35.2, 31.1(3C); IR vmax 3030, 1660, 1601, 1403, 1375, 860, 750, $700 \mathrm{~cm}^{-1}$; HRMS (EI) $\mathrm{m} / \mathrm{z}$ calcd for $\mathrm{C}_{21} \mathrm{H}_{24} \mathrm{NO}_{2}[\mathrm{M}+\mathrm{H}]^{+}$322.1802, found 322.1808.

\subsection{8. [1,1'-Biphenyl]-4-yl(5-benzyl-4,5-dihydroisoxazol-3-yl)methanone (3h)}

The compound was prepared following the general procedure. Yield $57 \% .{ }^{1} \mathrm{H}$ NMR $\left(400 \mathrm{MHz}, \mathrm{CDCl}_{3}\right) \delta 8.25-8.19(\mathrm{~m}, 2 \mathrm{H}), 7.69-7.68(\mathrm{~m}, 1 \mathrm{H}), 7.67(\mathrm{~m}, 1 \mathrm{H}), 7.65(\mathrm{~m}, 1 \mathrm{H})$, $7.63(\mathrm{~m}, 1 \mathrm{H}), 7.49(\mathrm{~m}, 1 \mathrm{H}), 7.47(\mathrm{~m}, 1 \mathrm{H}), 7.46(\mathrm{~m}, 1 \mathrm{H}), 7.43(\mathrm{~m}, 1 \mathrm{H}), 7.35-7.33(\mathrm{~m}, 1 \mathrm{H})$, $7.32(\mathrm{~m}, 1 \mathrm{H}), 7.30-7.28(\mathrm{~m}, 2 \mathrm{H}), 5.08(\mathrm{ddt}, J=10.8,7.9,6.3 \mathrm{~Hz}, 1 \mathrm{H}), 3.38(\mathrm{dd}, J=17.6$, $10.8 \mathrm{~Hz}, 1 \mathrm{H}), 3.17-3.09(\mathrm{~m}, 2 \mathrm{H}), 2.98(\mathrm{dd}, J=14.0,6.5 \mathrm{~Hz}, 1 \mathrm{H}) ;{ }^{13} \mathrm{C}$ NMR $(101 \mathrm{MHz}, \mathrm{CDCl} 3)$ $\delta$ 186.0, 157.9, 146.4, 139.9, 136.2, 134.6, 131.0(2C), 129.6(2C), 129.1(2C), 128.8(2C), 128.5(2C), 127.5(2C), 127.1(2C), 83.7, 41.1, 38.5; IR vmax 3029, 1655, 1648, 1401, 1362, 842, 746, $693 \mathrm{~cm}^{-1}$; HRMS (EI) $m / z$ calcd for $\mathrm{C}_{23} \mathrm{H}_{20} \mathrm{NO}_{2}[\mathrm{M}+\mathrm{H}]^{+} 342.1489$, found 342.1483. These data are consistent with the data reported in the literature [11].

\subsubsection{Methyl 5-benzyl-4,5-dihydroisoxazole-3-carboxylate (3i)}

The compound was prepared following the general procedure. Yield $30 \% .{ }^{1} \mathrm{H}$ NMR $\left(400 \mathrm{MHz}, \mathrm{CDCl}_{3}\right) \delta 7.32(\mathrm{~m}, 2 \mathrm{H}), 7.26(\mathrm{~m}, 1 \mathrm{H}), 7.22(\mathrm{~m}, 2 \mathrm{H}), 5.06(\mathrm{ddd}, J=14.7,10.9,6.7 \mathrm{~Hz}$, $1 \mathrm{H}), 3.86(\mathrm{~s}, 3 \mathrm{H}), 3.18(\mathrm{dd}, J=17.7,10.9 \mathrm{~Hz}, 1 \mathrm{H}), 3.11(\mathrm{dd}, J=14.0,6.1 \mathrm{~Hz}, 1 \mathrm{H}), 2.94(\mathrm{dd}$, $J=14.8,5.3 \mathrm{~Hz}, 1 \mathrm{H}), 2.92-2.85(\mathrm{~m}, 1 \mathrm{H}) ;{ }^{13} \mathrm{C}$ NMR $(101 \mathrm{MHz}, \mathrm{CDCl} 3) \delta 161.2,151.3,136.0$, 129.5(2C), 128.8(2C), 127.1, 84.5, 52.8, 40.8, 37.9; IR vmax 3418, 3032, 1717, 1584, 1449, 1366, 
$1265,1126,949,746,702,582 \mathrm{~cm}^{-1}$; HRMS (EI) $m / z$ calcd for $\mathrm{C}_{12} \mathrm{H}_{14} \mathrm{NO}_{3}[\mathrm{M}+\mathrm{H}]^{+} 220.0974$, found 220.0980 .

\subsubsection{0. (5-(2-Methylbenzyl)-4,5-dihydroisoxazol-3-yl)(phenyl)methanone (5a)}

The compounds was prepared following the general procedure as isomers. Yield $71 \% .{ }^{1} \mathrm{H}$ $\operatorname{NMR}\left(400 \mathrm{MHz}, \mathrm{CDCl}_{3}\right)$ 8 8.30-8.23 (m, 2H), 7.66 (m, 1H), 7.57-7.49 (m, 2H), 7.30-7.23 (m, 4H), 5.19-5.08 (m, 1H), $3.42(\mathrm{ddd}, J=17.5,10.7,0.9 \mathrm{~Hz}, 1 \mathrm{H}), 3.22(\mathrm{dt}, J=12.6,7.5 \mathrm{~Hz}, 2 \mathrm{H}), 2.99(\mathrm{dd}$, $J=14.3,6.7 \mathrm{~Hz}, 1 \mathrm{H}), 2.44(\mathrm{~s}, 3 \mathrm{H}) ;{ }^{13} \mathrm{C}$ NMR $(101 \mathrm{MHz}, \mathrm{CDCl} 3) \delta 186.5,157.9,136.6,135.9$, 134.6, 133.6, 130.6, 130.4(2C), 130.0, 128.4(2C), 127.1, 126.3, 82.9, 38.6, 38.1, 19.8; IR vmax 3028, 2940, 1660, 1570, 750, $690 \mathrm{~cm}^{-1}$; HRMS (EI) $m / z$ calcd for $\mathrm{C}_{18} \mathrm{H}_{18} \mathrm{NO}_{2}[\mathrm{M}+\mathrm{H}]^{+} 280.1332$, found 280.1335 .

\subsubsection{1. (5-(3-Methylbenzyl)-4,5-dihydroisoxazol-3-yl)(phenyl)methanone (5b)}

The compound was prepared following the general procedure. Yield $66 \% .{ }^{1} \mathrm{H}$ NMR $\left(400 \mathrm{MHz}, \mathrm{CDCl}_{3}\right) \delta 8.15(\mathrm{~d}, J=7.2 \mathrm{~Hz}, 2 \mathrm{H}), 7.59(\mathrm{~m}, 1 \mathrm{H}), 7.47(\mathrm{~m}, 2 \mathrm{H}), 7.22$ $(\mathrm{m}, 1 \mathrm{H}), 7.09(\mathrm{~m}, 3 \mathrm{H}), 5.12-5.00(\mathrm{~m}, 1 \mathrm{H}), 3.35(\mathrm{dd}, J=17.5,10.8,1 \mathrm{H}), 3.17-3.04(\mathrm{~m}, 2 \mathrm{H})$, 2.97-2.87 (m, 1H), $2.36(\mathrm{~s}, 3 \mathrm{H}) ;{ }^{13} \mathrm{C}$ NMR (101 MHz, CDCl3) $\delta 186.6,157.8,138.4,136.1$, $135.9,133.7,130.4(2 \mathrm{C}), 130.4,128.7,128.5(2 \mathrm{C}), 127.9,126.6,83.8,40.9,38.5,21.5$; IR vmax $3048,2908,1661,1581,862,750,691 \mathrm{~cm}^{-1}$; HRMS (EI) $m / z$ calcd for $\mathrm{C}_{18} \mathrm{H}_{18} \mathrm{NO}_{2}[\mathrm{M}+\mathrm{H}]^{+}$ 280.1332 , found 280.1340 .

\subsubsection{2. (5-(4-Methylbenzyl)-4,5-dihydroisoxazol-3-yl)(phenyl)methanone (5c)}

The compound was prepared following the general procedure. Yield $64 \% .{ }^{1} \mathrm{H}$ NMR $\left(400 \mathrm{MHz}, \mathrm{CDCl}_{3}\right) \delta 8.16-8.10(\mathrm{~m}, 2 \mathrm{H}), 7.59(\mathrm{~m}, 1 \mathrm{H}), 7.46(\mathrm{~m}, 2 \mathrm{H}), 7.16(\mathrm{~m}, 4 \mathrm{H}), 5.05(\mathrm{dd}$, $J=11.0,7.8 \mathrm{~Hz}, 1 \mathrm{H}), 3.35(\mathrm{dd}, J=17.6,10.8 \mathrm{~Hz}, 1 \mathrm{H}), 3.15-3.04(\mathrm{~m}, 2 \mathrm{H}), 2.93(\mathrm{dd}, J=14.0$, $6.6 \mathrm{~Hz}, 1 \mathrm{H}), 2.34(\mathrm{~s}, 3 \mathrm{H}) ;{ }^{13} \mathrm{C}$ NMR $(101 \mathrm{MHz}, \mathrm{CDCl} 3) \delta 186.7,157.9,136.8,136.1,133.8$, 133.2, 130.5(2C), 129.6(2C), 129.6(2C), 128.6(2C), 83.9, 40.7, 38.5, 21.3; IR vmax 3039, 2909, 1710, 1609, 822, 741, $680 \mathrm{~cm}^{-1}$; HRMS (EI) $\mathrm{m} / z$ calcd for $\mathrm{C}_{18} \mathrm{H}_{18} \mathrm{NO}_{2}[\mathrm{M}+\mathrm{H}]^{+}$280.1332, found 280.1339 .

\subsubsection{3. (5-(4-Methoxybenzyl)-4,5-dihydroisoxazol-3-yl)(phenyl)methanone (5d)}

The compound was prepared following the general procedure. Yield $72 \% .{ }^{1} \mathrm{H}$ NMR $\left(400 \mathrm{MHz}, \mathrm{CDCl}_{3}\right) \delta 8.12(\mathrm{~m}, 2 \mathrm{H}), 7.58(\mathrm{~m}, 1 \mathrm{H}), 7.45(\mathrm{~m}, 2 \mathrm{H}), 7.18(\mathrm{~m}, 2 \mathrm{H}), 6.86(\mathrm{~m}, 2 \mathrm{H})$, $5.08-4.96(\mathrm{~m}, 1 \mathrm{H}), 3.78(\mathrm{~s}, 3 \mathrm{H}), 3.34(\mathrm{dd}, J=17.6,10.8 \mathrm{~Hz}, 1 \mathrm{H}), 3.14-2.99(\mathrm{~m}, 2 \mathrm{H}), 2.91(\mathrm{dd}$, $J=14.1,6.4 \mathrm{~Hz}, 1 \mathrm{H}) ;{ }^{13} \mathrm{C}$ NMR $(101 \mathrm{MHz}, \mathrm{CDCl} 3) \delta 186.6,158.7,157.8,135.9,133.6$, 130.6(2C), 130.4(2C), 128.4(2C), 128.1, 114.2(2C), 83.8, 55.3, 40.0, 38.3; IR vmax 3050, 2850, 1670, 1580, 820, 750, $691 \mathrm{~cm}^{-1}$; HRMS (EI) $\mathrm{m} / \mathrm{z}$ calcd for $\mathrm{C}_{18} \mathrm{H}_{18} \mathrm{NO}_{3}[\mathrm{M}+\mathrm{H}]^{+} 296.1281$, found 296.1285 .

\subsubsection{4. (5-Octyl-4,5-dihydroisoxazol-3-yl)(phenyl)methanone (5e)}

The compound was prepared following the general procedure. Yield $89 \% .{ }^{1} \mathrm{H}$ NMR $\left(400 \mathrm{MHz}, \mathrm{CDCl}_{3}\right) \delta 8.25-8.12(\mathrm{~m}, 2 \mathrm{H}), 7.58(\mathrm{~m}, 1 \mathrm{H}), 7.46(\mathrm{~m}, 2 \mathrm{H}), 4.79(\mathrm{ddt}, J=10.9,8.4$, $6.6 \mathrm{~Hz}, 1 \mathrm{H}), 3.39(\mathrm{dd}, J=17.4,10.9 \mathrm{~Hz}, 1 \mathrm{H}), 3.00(\mathrm{dd}, J=17.4,8.5 \mathrm{~Hz}, 1 \mathrm{H}), 1.79(\mathrm{~m}, 1 \mathrm{H})$, 1.69-1.57 (m, 1H), 1.45-1.19 (m, 12H), $0.88(\mathrm{~m}, 3 \mathrm{H}) ;{ }^{13} \mathrm{C} \mathrm{NMR}(101 \mathrm{MHz}, \mathrm{CDCl} 3) \delta 186.7$, 157.9, 136.0, 133.6, 130.5(2C), 128.5(2C), 83.7, 38.9, 35.3, 31.9, 29.6, 29.5, 29.3, 25.4, 22.8, 14.2; IR vmax 3062, 2948, 1635, 1541, 760, $710 \mathrm{~cm}^{-1}$; HRMS (EI) $\mathrm{m} / z$ calcd for $\mathrm{C}_{18} \mathrm{H}_{26} \mathrm{NO}_{2}$ $[\mathrm{M}+\mathrm{H}]^{+} 288.1958$, found 288.1961. These data are consistent with the data reported in the literature [11].

\subsubsection{5. (5-(Chloromethyl)-4,5-dihydroisoxazol-3-yl)(phenyl)methanone (5f)}

The compound was prepared following the general procedure. Yield $83 \% .{ }^{1} \mathrm{H}$ NMR $\left(400 \mathrm{MHz}, \mathrm{CDCl}_{3}\right)$ 8 8.24-8.14 (m, 2H), 7.66-7.55 (m, 1H), 7.53-7.44 (m, 2H), 5.05 (dddd, $J=11.1,7.1,5.7,4.5 \mathrm{~Hz}, 1 \mathrm{H}), 3.76-3.62(\mathrm{~m}, 2 \mathrm{H}), 3.50(\mathrm{dd}, J=17.9,11.1 \mathrm{~Hz}, 1 \mathrm{H}), 3.36(\mathrm{dd}$, $J=17.9,7.1 \mathrm{~Hz}, 1 \mathrm{H}) ;{ }^{13} \mathrm{C}$ NMR $(101 \mathrm{MHz}, \mathrm{CDCl} 3) \delta 186.0,157.5,135.7,133.9,130.5(2 \mathrm{C})$, 
128.6(2C), 81.2, 45.1, 37.7; IR vmax 3025, 1660, 1580, $700 \mathrm{~cm}^{-\mathrm{m}}$; HRMS (EI) $\mathrm{m} / \mathrm{z}$ calcd for $\mathrm{C}_{11} \mathrm{H}_{11} \mathrm{NO}_{2} \mathrm{Cl}[\mathrm{M}+\mathrm{H}]^{+} 224.0473$, found 224.0466. These data are consistent with the data reported in the literature [11].

3.2.16. (3a,4,5,6,7,7a-Hexahydrobenzo[d]isoxazol-3-yl)(phenyl)methanone (5g)

The compound was prepared following the general procedure. Yield $54 \% .{ }^{1} \mathrm{H}$ NMR $\left(400 \mathrm{MHz} \mathrm{CDCl}_{3}\right) \delta 8.18(\mathrm{~m}, 2 \mathrm{H}), 7.61-7.56(\mathrm{~m}, 1 \mathrm{H}), 7.47(\mathrm{~m}, 2 \mathrm{H}), 4.59(\mathrm{dt}, J=7.9,3.9 \mathrm{~Hz}$, $1 \mathrm{H}), 3.46-3.38(\mathrm{~m}, 1 \mathrm{H}), 2.20(\mathrm{dd}, J=15.2,3.5 \mathrm{~Hz}, 1 \mathrm{H}), 2.11-2.01(\mathrm{~m}, 1 \mathrm{H}), 1.82(\mathrm{tt}, J=15.3$, $4.7 \mathrm{~Hz}, 1 \mathrm{H}), 1.65-1.52$ (m, 3H), 1.35-1.24 (m, 2H); ${ }^{13} \mathrm{C}$ NMR (101 MHz, CDCl3) $\delta ~ 187.0$, 163.8, 136.4, 133.6, 130.4(2C), 128.5(2C), 82.3, 44.3, 25.6, 25.0, 21.7, 19.9; IR vmax 3060, 2940, $1660,1550,747,704 \mathrm{~cm}^{-\mathrm{m}}$; HRMS (EI) $\mathrm{m} / z$ calcd for $\mathrm{C}_{14} \mathrm{H}_{16} \mathrm{NO}_{2}[\mathrm{M}+\mathrm{H}]^{+} 230.1176$, found 230.1182. These data are consistent with the data reported in the literature [11].

\subsubsection{Ethyl 3-benzoyl-4,5-dihydroisoxazole-5-carboxylate (7a)}

The compound was prepared following the general procedure. Yield $68 \% .{ }^{1} \mathrm{H}$ NMR (400 MHz, $\left.\mathrm{CDCl}_{3}\right) \delta 8.33-8.28(\mathrm{~m}, 2 \mathrm{H}), 7.71-7.65(\mathrm{~m}, 1 \mathrm{H}), 7.59-7.51(\mathrm{~m}, 2 \mathrm{H}), 7.43(\mathrm{~s}, 1 \mathrm{H})$, $4.48(\mathrm{q}, J=7.1 \mathrm{~Hz}, 2 \mathrm{H}), 1.44(\mathrm{t}, J=7.1 \mathrm{~Hz}, 3 \mathrm{H}) ;{ }^{13} \mathrm{C} \mathrm{NMR}(101 \mathrm{MHz}, \mathrm{CDCl} 3) \delta 184.7$, $162.3,161.3,156.4,135.3,134.6,130.9(2 C), 128.9(2 C), 110.2,62.8,14.3$; IR vmax 3058, 2945, $1655,1545,740,702 \mathrm{~cm}^{-\mathrm{m}}$; HRMS (EI) $\mathrm{m} / z$ calcd for $\mathrm{C}_{13} \mathrm{H}_{14} \mathrm{NO}_{2}[\mathrm{M}+\mathrm{H}]^{+} 248.0917$, found 248.0912 .

\subsubsection{8. (5-Butylisoxazol-3-yl)(phenyl)methanone (7b)}

The compound was prepared following the general procedure. Yield $64 \% .{ }^{1} \mathrm{H}$ NMR $\left(400 \mathrm{MHz}, \mathrm{CDCl}_{3}\right) \delta 8.29(\mathrm{~m}, 2 \mathrm{H}), 7.61(\mathrm{~m}, 1 \mathrm{H}), 7.49(\mathrm{~m}, 2 \mathrm{H}), 6.51(\mathrm{~ms}, 1 \mathrm{H}), 2.82(\mathrm{t}, J=7.6 \mathrm{~Hz}$, $2 \mathrm{H}), 1.73(\mathrm{dt}, J=15.2,7.5 \mathrm{~Hz}, 2 \mathrm{H}), 1.42(\mathrm{dq}, J=14.6,7.4 \mathrm{~Hz}, 2 \mathrm{H}), 0.95(\mathrm{t}, J=7.4 \mathrm{~Hz}, 3 \mathrm{H})$; ${ }^{13} \mathrm{C}$ NMR (101 MHz, CDCl3) $\delta 186.2,174.8,161.9,135.9,133.9,130.7(2 \mathrm{C}), 128.6(2 \mathrm{C}), 101.7$, 29.6, 26.4, 22.3, 13.8. IR $v \max 3075,2950,2875,1670,1590,740,690 \mathrm{~cm}^{-\mathrm{m}}$; HRMS (EI) $\mathrm{m} / \mathrm{z}$ calcd for $\mathrm{C}_{14} \mathrm{H}_{16} \mathrm{NO}_{2}[\mathrm{M}+\mathrm{H}]^{+} 230.1176$, found 230.1171. These data are consistent with the data reported in the literature [11].

\section{Conclusions}

Isoxazolines and isoxazoles are biologically active molecules. The development and improvement of syntheses directed towards isoxazolines and isoxazoles is a continuing pursuit. Herein, we have developed an effective cycloaddition of various $\alpha$-nitroketones with alkenes or alkynes by using the cheap base chloramine-T. The low cost and ease of handling of this moderate base are its outstanding properties. The cycloaddition described in this study is an integrated approach for synthesizing isoxazolines and isoxazoles. We are currently investigating other ways to integrate isoxazolines.

Supplementary Materials: The following are available online. The Supplementary Materials contain experimental protocols, analytical data for products and NMR spectra.

Author Contributions: X.P., Y.M. and J.W.: conceptualization; X.P. and X.X.: methodology; X.P., X.X., Y.Z. and K.Z.: data analysis; X.P., Y.M., X.L. and Y.L.: original manuscript writing; X.P., X.Y. and J.W.: revision and supervision. All authors have read and agreed to the published version of the manuscript.

Funding: This study was supported by The Open Project of Key Laboratory of Xinjiang Phytomedicine Resource and Utilization, Ministry of Education (grant numbers 20150203 and XPRU202004); National Science and Technology Major Projects for New Drug Development of China (grant number 2018ZX09735-005); and Youth Innovative Talent Cultivation Projects of Shihezi University (grant number CXPY202005).

Institutional Review Board Statement: Not applicable.

Informed Consent Statement: Not applicable. 
Data Availability Statement: The data presented in this study are available in the article and Supplementary Materials.

Acknowledgments: We would like to thank Lei Liu and Jinchuan Zhou (Shandong University) for their help with the experimental design and writing of the manuscript. Liang Guo and Pengyu Dai (Traditional Chinese Medicine University of Guangzhou) are acknowledged for assistance with NMR.

Conflicts of Interest: The authors declare no conflict of interest.

\section{References}

1. Chen, C.-H.; Wu, Q.-Y.; Wei, C.; Liang, C.; Su, G.-F.; Mo, D.-L. Iron(iii)-catalysed selective N-O bond cleavage to prepare tetrasubstituted pyridines and 3,5-disubstituted isoxazolines from $\mathrm{N}$-vinyl- $\alpha, \beta$-unsaturated ketonitrones. Green Chem. 2018, 20 , 2722-2729. [CrossRef]

2. Zhang, L.H.; Chung, J.C.; Costello, T.D.; Valvis, I.; Ma, P.; Kauffman, S.; Ward, R. The Enantiospecific Synthesis of an Isoxazoline. A RGD Mimic Platelet GPIIb/IIIa Antagonist. J. Org. Chem. 1997, 62, 2466-2470. [CrossRef]

3. Sun, R.; Li, Y.; Xiong, L.; Liu, Y.; Wang, Q. Design, Synthesis, and Insecticidal Evaluation of New Benzoylureas Containing Isoxazoline and Isoxazole Group. J. Agric. Food Chem. 2011, 59, 4851-4859. [CrossRef]

4. Ismail, T.; Shafi, S.; Singh, S.; Sidiq, T.; Khajuria, A.; Rouf, A.; Yadav, M.; Saikam, V.; Singh, P.P.; Alam, M.S.; et al. Synthesis and immunopotentiating activity of novel isoxazoline functionalized coumarins. Eur. Med. Chem. 2016, 123, 90-104. [CrossRef]

5. Kamal, A.; Reddy, J.S.; Ramaiah, M.J.; Dastagiri, D.; Bharathi, E.V.; Azhar, M.A.; Sultana, F.; Pushpavalli, S.N.C.V.L.; Pal-Bhadra, M.; Juvekar, A.; et al. Design, synthesis and biological evaluation of 3,5-diaryl-isoxazoline/isoxazole-pyrrolobenzodiazepine conjugates as potential anticancer agents. Eur. J. Med. Chem. 2010, 45, 3924-3937. [CrossRef]

6. Kim, B.H.; Chung, Y.J.; Ryu, E.J. Synthesis of $\alpha$-hydroxy ketomethylene dipeptide isosteres. Tetrahedron Lett. 1993, 34, 8465-8468.

7. Curran, D.P. Reduction of. DELTA. 2-isoxazolines. 3. Raney nickel catalyzed formation of. beta.-hydroxy ketones. J. Am. Chem. Soc. 1983, 105, 5826-5833. [CrossRef]

8. Curran, D.P.; Kim, B.H. Reduction of 4,5-Dihydro-1,2-oxazoles ( $\Delta$-Isoxazolines); A Cycloadditive Approach to 2-Alkenyl Ketones. Synthesis 1986, 4, 312-315. [CrossRef]

9. Kozikowski, A.P.; Stein, P.D. The INOC route to carbocyclics: A formal total synthesis of ( \pm )-sarkomycin. J. Am. Chem. Soc. 1982, 104, 4023-4024. [CrossRef]

10. Muri, D.; Carreira, E.M. Stereoselective Synthesis of Erythronolide A via Nitrile Oxide Cycloadditions and Related Studies. J. Org. Chem. 2009, 74, 8695-8712. [CrossRef]

11. Choe, H.; Cho, H.; Ko, H.-J.; Lee, J. Total Synthesis of (+)-Pochonin D and (+)-Monocillin II via Chemo-and Regioselective Intramolecular Nitrile Oxide Cycloaddition. Org. Lett. 2017, 19, 6004-6007. [CrossRef]

12. Choe, H.; Pham, T.T.; Lee, J.Y.; Latif, M.; Park, H.; Kang, Y.K.; Lee, J. Remote Stereoinductive Intramolecular Nitrile Oxide Cycloaddition: Asymmetric Total Synthesis and Structure Revision of (-)-11ß-Hydroxycurvularin. J. Org. Chem. 2016, 81, 2612-2617. [CrossRef]

13. Gothelf, K.V.; Jørgensen, K.A. Asymmetric 1,3-Dipolar Cycloaddition Reactions. Chem. Rev. 1998, 98, 863-910. [CrossRef]

14. Collington, E.W.; Knight, J.G.; Wallis, C.J.; Warren, S. Regiospecific synthesis of (E) unsaturated 3,5-dialkyl-isoxazoles and derived leukotriene analogues using phosphine oxides. Tetrahedron Lett. 1989, 30, 877-880. [CrossRef]

15. Nazarenko, K.G.; Shvidenko, K.V.; Pinchuk, A.M.; Tolmachev, A.A. Synthesis of 7-Amino-1-nitro-2-heptanone Derivatives. Synth Commun. 2003, 33, 4241-4252. [CrossRef]

16. Shimizu, T.; Hayashi, Y.; Teramura, K. The Reaction of Primary Nitro Compounds with Dipolarophiles in the Presence of p-Toluenesulfonic Acid. Bull. Chem. Soc. Jpn. 1984, 57, 2531-2534. [CrossRef]

17. Wade, P.A.; Amin, N.V.; Yen, H.K.; Price, D.T.; Huhn, G.F. Acid-catalyzed nitronate cycloaddition reactions. Useful syntheses and simple transformations of 3-acyl- and 3-alkenylisoxazolines. J. Org. Chem. 1984, 49, 4595-4601. [CrossRef]

18. Itoh, K.-i.; Aoyama, T.; Satoh, H.; Fujii, Y.; Sakamaki, H.; Takido, T.; Kodomari, M. Application of silica gel-supported polyphosphoric acid $\left(\mathrm{PPA} / \mathrm{SiO}_{2}\right)$ as a reusable solid acid catalyst to the synthesis of 3-benzoylisoxazoles and isoxazolines. Tetrahedron Lett. 2011, 52, 6892-6895. [CrossRef]

19. Yavari, I.; Piltan, M.; Moradi, L. Synthesis of pyrrolo[2,1-a]isoquinolines from activated acetylenes, benzoylnitromethanes, and isoquinoline. Tetrahedron 2009, 65, 2067-2071. [CrossRef]

20. Machetti, F.; Cecchi, L.; Trogu, E.; De Sarlo, F. Isoxazoles and Isoxazolines by 1,3-Dipolar Cycloaddition: Base-Catalysed Condensation of Primary Nitro Compounds with Dipolarophiles. Eur. J. Org. Chem. 2007, 2007, 4352-4359. [CrossRef]

21. Cecchi, L.; De Sarlo, F.; Machetti, F. Synthesis of 4,5-Dihydroisoxazoles by Condensation of Primary Nitro Compounds with Alkenes by Using a Copper/Base Catalytic System. Chem. A Eur. J. 2008, 14, 7903-7912. [CrossRef]

22. Umemoto, N.; Imayoshi, A.; Tsubaki, K. Nitrile oxide cycloaddition reactions of alkenes or alkynes and nitroalkanes substituted with O-alkyloxime groups convertible to various functional groups. Tetrahedron Lett. 2020, 61, 152213-152216. [CrossRef]

23. Lee, J.J.; Kim, J.; Jun, Y.M.; Lee, B.M.; Kim, B.H. Indium-mediated one-pot synthesis of benzoxazoles or oxazoles from 2nitrophenols or 1-aryl-2-nitroethanones. Tetrahedron 2009, 65, 8821-8831. [CrossRef] 